\title{
Parcours de vie et âges des transitions en Belgique : vers une déstandardisation des âges à l'entrée et au départ du marché du travail ?
}

\section{Life course and ages of transitions in Belgium : Towards a de-standardization of ages at entry and exit from the labor market?}

\author{
Jean-Paul Sanderson
}

Volume 44, numéro 1, printemps 2015

URI : https://id.erudit.org/iderudit/1032149ar

DOI : https://doi.org/10.7202/1032149ar

Aller au sommaire du numéro

Éditeur(s)

Association des démographes du Québec

ISSN

1705-1495 (numérique)

Découvrir la revue

Citer cet article

Sanderson, J.-P. (2015). Parcours de vie et âges des transitions en Belgique : vers une déstandardisation des âges à l'entrée et au départ du marché du travail ? Cahiers québécois de démographie, 44(1), 65-87.

https://doi.org/10.7202/1032149ar

\section{Résumé de l'article}

Depuis plusieurs décennies, on assiste à une transformation profonde des parcours professionnels. L'entrée dans la vie active est de plus en plus tardive et complexe, tandis que les départs du marché du travail sont devenus très précoces, en Europe et particulièrement en Belgique, au point d'inquiéter les décideurs politiques et de les inciter à intervenir. L'analyse de cette double transformation a amené différents auteurs à formuler l'hypothèse d'une déstandardisation des parcours de vie à partir des années 1960. À partir de données rétrospectives sur la Belgique, cet article entend tester l'hypothèse d'une déstandardisation des âges à l'entrée et à la sortie du marché du travail au cours des années 1965-2001.
Tous droits réservés @ Association des démographes du Québec, 2015
Ce document est protégé par la loi sur le droit d'auteur. L'utilisation des services d’Érudit (y compris la reproduction) est assujettie à sa politique d'utilisation que vous pouvez consulter en ligne. 


\title{
Parcours de vie et âges des transitions en Belgique: vers une déstandardisation des âges à l'entrée et au départ du marché du travail?
}

\author{
JEAN-PAUL SANDERSON*
}

\begin{abstract}
Depuis plusieurs décennies, on assiste à une transformation profonde des parcours professionnels. L'entrée dans la vie active est de plus en plus tardive et complexe, tandis que les départs du marché du travail sont devenus très précoces, en Europe et particulièrement en Belgique, au point d'inquiéter les décideurs politiques et de les inciter à intervenir. L'analyse de cette double transformation a amené différents auteurs à formuler l'hypothèse d'une déstandardisation des parcours de vie à partir des années 1960. À partir de données rétrospectives sur la Belgique, cet article entend tester l'hypothèse d'une déstandardisation des âges à l'entrée et à la sortie du marché du travail au cours des années 1965-2001.
\end{abstract}

English abstract p. 87

\section{INTRODUCTION}

$\mathrm{D}$ epuis le milieu du $19^{\mathrm{e}}$ siècle et l'avènement de la société industrielle, la valeur structurante des sociétés européennes est la valeur «travail» (Royer, 2002). Les analyses menées dans le cadre du paradigme des parcours de vie (Elder, Kirkpatrick et Crosnoe, 2003) mettent en évidence cette structuration autour de cette valeur centrale avec des vies individuelles articulées autour de la période d'activité professionnelle (Guillemard, 2004) : les parcours individuels sont divisés selon un modèle ternaire (enfance $=$ apprentissage, vie adulte $=$ activité professionnelle et construction familiale, retraite $=$ vieillesse, temps de repos) (Roussel et Girard, 1982). La Belgique n’a pas échappé à ces mécanismes et on y observe également une structuration des parcours de vie autour

* Centre de Recherche en Démographie et Sociétés, Université catholique de Louvain Louvain-la-Neuve, Belgique (Jean-paul.sanderson@uclouvain.be) 
de la période de vie active, avec un risque de disqualification voire d'exclusion sociale pour ceux qui sortent du modèle (Burnay, 2002).

Aujourd'hui, le rapport au monde du travail et à l'emploi se transforment (Royer, 2002). La fragilisation des parcours professionnels depuis la crise des années 1970 a fait perdre au travail son caractère de vecteur d'intégration universel, certains demeurant exclus du marché du travail.

Parallèlement, les pays européens, dont la Belgique, connaissent ce que les démographes qualifient de deuxième transition démographique, où l'individualisation accrue et le besoin de se réaliser soi-même conduisent à un modèle où le dessein individuel semble prendre le pas sur un modèle plus familial: de nouvelles formes d'unions et de familles se développent (famille monoparentale, couples cohabitants, unions non cohabitantes...) avec des résonnances dans la vie professionnelle, où les trajectoires deviennent moins linéaires (Lesthaeghe et Neels, 2002). Ces transformations et cette fragilisation ont comme conséquence des parcours professionnels plus chaotiques et donc moins prévisibles. Ces éléments ont amené à la formulation d'une hypothèse de déstandardisation des trajectoires individuelles (Kholi, 1986) s'exprimant par une complexification des séquences, avec notamment l'alternance de périodes d'emploi et de chômage (Widmer et Ritschard, 2013), mais aussi par une déchronologisation des seuils auxquels les passages d'une étape à l'autre sont vécus.

Dans ce cadre, l'hypothèse généralement admise est celle d'un $20^{\mathrm{e}}$ siècle marqué par deux étapes : une période de standardisation des âges-clefs marquant le passage d'une étape à l'autre (passage de l'enfanceadolescence à la vie adulte et passage à la retraite) du fait de leur institutionnalisation, période s'achevant dans les années 1960, puis une déstandardisation et une désinstitutionnalisation progressive des séquences et des âges clefs depuis lors (Kholi, 1986).

L'objectif de cet article est de s'interroger sur ce processus de déstandardisation des âges auxquels sont vécues ces transitions à partir de l'exemple de la Belgique. L'hypothèse testée est celle d'une déstandardisation des âges à l'entrée et au départ du marché du travail. Si le report de l'âge à l'entrée sur le marché du travail et le rajeunissement des départs anticipés du marché du travail semblent clairement établis (Sanderson, Eggerickx et Burnay, 2012; Aubert, 2012), une déstandardisation suppose l'accroissement de la diversité des situations. Cette diversification devrait se traduire statistiquement dans les indicateurs de dispersion et notamment dans les valeurs des écarts-types autour de la moyenne. 


\section{CADRE THÉORIQUE}

Le paradigme des parcours de vie développé par Glen Elder (1998) propose d'analyser les vies individuelles dans leur déroulement temporel et dans leur contexte sociohistorique (Cavali, 2007). Cette approche repose sur quatre principes heuristiques (Hicks, 2013, p. 306) :

(1) les expériences quotidiennes constituent une trame qui commence à la naissance et se poursuit jusqu'à la mort ; (2) les parcours de vie se déroulent selon de multiples dimensions interconnectées; (3) des liens sociaux se créent tout au long de notre parcours de vie et de celui des autres; et (4) divers contextes géographiques et nationaux façonnent les parcours de vie et sont à leur tour façonnés par eux.

Gaudet et Turcotte (2013) ajoutent un $5^{\mathrm{e}}$ principe, celui de l'autonomie des individus, à savoir leur capacité d'action sur leurs conditions de vie, et ils complètent le 4 e principe en y intégrant le contexte historique dans lequel se déroulent les vies individuelles.

Les travaux menés dans ce cadre se sont focalisés sur les $19^{\mathrm{e}}$ et $20^{\mathrm{e}}$ siècles et ont mis en évidence différents processus à l'œuvre au cours de cette période. On parle ainsi de standardisation, de déstandardisation, d'institutionnalisation, de désinstitutionnalisation. Brückner et Mayer (2005, p. 32-33, notre traduction) définissent ces termes de la manière suivante :

L'institutionnalisation des parcours de vie se réfère au processus par lequel les règles normatives, juridiques ou organisationnelles définissent l'organisation sociale et temporelle des vies humaines. Elle peut se référer à des étapes ou des états dans la vie qui peuvent être formellement ou informellement décrétés comme le mariage, l'éducation et la retraite. Il peut également se référer à des événements et des transitions comme la fin de la scolarité, l'entrée et la sortie du marché du travail, ou les âges légaux à pension.

Inversement, la désinstitutionnalisation signifierait alors que les états, les étapes, les événements et les transitions, qui auparavant étaient clairement différenciés, sont réintégrés ou fusionnés.

La standardisation des parcours de vie se réfère aux processus par lesquels des états ou des événements spécifiques et les séquences dans lesquelles ils se produisent deviennent plus universel pour les populations données ou que leur synchronisation devient plus uniforme. Un exemple d'un modèle de cycle de vie hautement 
normalisé serait, par exemple, si tous les travailleurs partaient à la retraite à 65 ans.

Inversement, déstandardisation signifierait que les étapes de la vie, les événements et leurs séquences peuvent devenir des expériences qui caractérisent soit une part de plus en plus petite d'une population ou se produisent à des âges plus dispersés et avec des durées plus dispersées.

Standardisation et institutionnalisation marqueraient une première étape qui s'achèverait avec les années 1960 (Kholi, 1986). Les effets conjugués de l'industrialisation, de l'amélioration du niveau de vie, de l'alphabétisation, etc., vont provoquer un relâchement des contraintes que faisaient peser la famille et la communauté sur les comportements individuels. Parallèlement, le déclin de la mortalité et son recul vers des âges élevés font passer d'un régime de mort imprévisible et omniprésente à un régime de durée de vie moins incertain. Les individus semblent s'émanciper du contrôle social exercé par les familles et les communautés tout en composant avec une intervention grandissante de l'État dans une politique des âges scandant notamment les parcours scolaire et professionnel (Sapin, Spini et Widmer, 2007).

Ainsi, en Belgique, les législations portant sur le travail des enfants (loi de 1889 interdisant le travail des enfants de moins de 12 ans) et sur la mise en place de l'obligation scolaire ${ }^{1}$ permettront de définir un âge minimum à l'entrée sur le marché du travail (Loriaux, 1999), institutionnalisant ce dernier et aboutissant à une standardisation progressive des comportements, la plupart des jeunes finissant leur scolarité et entrant sur le marché du travail entre 14 et 18 ans.

Parallèlement, le départ du marché du travail va lui aussi être institutionnalisé, par le biais des législations sur la retraite, à l'exemple de la France (Feller, 2005). La Belgique tardera quelque peu et il faut attendre les années 1920 pour que soit instauré un système de retraite universelle et obligatoire pour les employés et les ouvriers. L’âge légal au départ du marché du travail est alors fixé à 65 ans pour les hommes et 60 ans pour les femmes. Des exceptions seront rapidement prévues pour certains métiers comme les mineurs ou les marins pour lesquels une durée d'exercice de la profession sera privilégiée plutôt qu'une limite d'âge. Toutefois, dans l'ensemble, au lendemain de la Seconde Guerre mondiale, la plupart des

1. L'obligation scolaire sera imposée aux enfants de 6 à 14 ans en 1914. En 1983, elle sera étendue aux adolescents de 14 à 18 ans. 
travailleurs quittent le marché du travail une fois atteint l'âge limite (65 ou 60 ans). On assiste alors à une standardisation de l'âge au départ du marché du travail (Vanthemsche, 1994).

Cette chronologisation des vies individuelles aboutit à l'instauration d'âges de passage d'un état vers un autre relativement précis. Parallèlement à une standardisation des âges auxquels s'effectuent les transitions, les trajectoires à l'intérieur de ces bornes semblent de plus en plus standardisées, normalisées (Cavalli, 2007). Elles en deviennent ainsi davantage prévisibles. Image d'Épinal d'un jeune terminant sa formation et quittant ses parents pour fonder son foyer et entamer une carrière unique au sein de la même entreprise (avec un contrat obtenu à l'issue de sa formation) tandis que l'épouse s'occupe du foyer et des enfants.

Cette image est mise à mal au tournant des années 1960. Deux explications complémentaires sont avancées pour comprendre ce changement. Cette période est marquée par de profonds bouleversements qui vont toucher aussi bien les sphères politiques et culturelles (par exemple mai 68) que la sphère privée, voire intime, avec notamment la révolution contraceptive (Oris, 2006) et par une montée de l'individualisation :

Les individus ne peuvent plus être considérés comme des sujets stables et immuables, mais plutôt comme des identités aux multiples visages et capables de se détourner d'une destinée non souhaitée. L'individu devient le producteur de son réseau relationnel et bénéficie d'une marge de manœuvre, il fait des choix. Il en résulte une hétérogénéité croissante des expériences de vie. (Carpentier et White, 2013, p. 282)

Parallèlement, un deuxième courant explicatif met en évidence le rôle de la crise économique des années 1970 :

La transformation du marché du travail, en particulier après la crise pétrolière de 1973, implique de plus en plus la prévalence de " mauvais » plutôt que de «bons » emplois avec les plus pauvres perspectives de carrière, une diminution des possibilités de mobilité, des salaires plus bas et moins d'avantages sociaux. Le nouveau contexte économique exacerbe également certains problèmes dans l'avancement du cycle de vie grâce à la fréquence accrue des conditions de récession qui produisent des pics de taux de chômage. (Macmillan, 2005, p. 17, notre traduction).

De fait, les transitions semblent plus laborieuses (Cavalli, 2007) et la fin des études et l'entrée dans la vie active ne correspondent plus nécessairement à 
l'accès au premier emploi. En Belgique, le taux de chômage général qui était de $2,37 \%$ en 1960, dépasse les $5 \%$ en 1975 et passe la barre des $15 \%$ dans la deuxième moitié des années 1980 (Vander Stricht, 1997). Parallèlement, la transition vers la retraite se transforme, la crise économique et la montée du chômage vont inciter le législateur belge à développer un ensemble de mesures visant à favoriser le départ anticipé du marché du travail des actifs âgés (chômeurs et travailleurs). L'objectif est de favoriser l'emploi des jeunes. Ce n'est qu'au tournant des années 2000 que ces mesures seront remises en question du fait de la prise de conscience de l'importance du vieillissement et de ses conséquences pour la sécurité sociale en Belgique (Moulaert, 2006). Cette seconde période serait donc marquée par la fragilisation des trajectoires professionnelles. Cette fragilisation va également affecter la sphère familiale, avec un accroissement du nombre de divorces et une baisse de la durée des unions.

Ainsi, le processus de standardisation céderait le pas à la déstandardisation et l'institutionnalisation à la désinstitutionnalisation (Bessin, 1999; Kholi, 1986). Le report progressif de l'entrée sur le marché du travail du fait de l'allongement des études, le développement de la formation continue (en cours de carrière), le rajeunissement de l'âge moyen au départ du marché du travail (Sanderson, Eggerickx et Burnay, 2012) et la diversification des modes de sorties du marché du travail (Burnay, 2002; Cavali, 2007) vont contribuer à l'émergence de limites floues entre les étapes, celles-ci survenant à des moments variables d'une personne à l'autre, traduisant ainsi l'individualisation accrue des comportements.

L'analyse de la littérature met en évidence un double effet de la déstandardisation à savoir la complexification et la diversification des parcours individuels, rendant ceux-ci de moins en moins prévisibles (Widmer, Ritschard et Müller, 2009; Widmer et Ritschard, 2013) et le « dérèglement de l'horloge sociale " avec une modification des limites et une diversification des âges auxquels les transitions sont vécues (Kholi, 1986). Citons à ce propos Schumacher, Spoorenberg et Forney (2006, p. 156) :

Les exigences du premier stade du modèle industriel du parcours de vie sont bouleversées; le calendrier et la synchronisation des principaux événements (premier emploi, départ du foyer parental, premier mariage et enfants) sont remis en question et les anciennes trajectoires normatives doivent être redéfinies ou même abandonnées. Les parcours de vie se déstandardisent et se pluralisent. Premièrement, les principaux événements du cours de la vie se déchronologisent. Ils se produisent en général plus tard et à un âge plus variable. 
Si la complexification des séquences semble bien démontrée (Widmer, Ritschard et Müller, 2009; Widmer et Ritschard, 2013), la déstandardisation des âges pose davantage question. Kohli (2007), citant les travaux de Schergers sur l'Allemagne de l'Ouest, montre que la déstandardisation des âges où surviennent les transitions clefs n'est pas toujours vérifiée. L'exemple développé porte précisément sur l'âge au départ du marché du travail.

Pour les hommes, l'âge médian a diminué de la première à la troisième cohorte [Il s'agit de cohortes Nées Entre 1910 et 1939], mais est resté constant depuis et l'intervalle interquartile ne montre presque pas de changement dans l'ensemble. Pour les femmes, l'âge médian est resté constant, alors que l'intervalle interquartile (qui est généralement plus grand que pour les hommes) a d'abord baissé puis augmenté à nouveau. Il n'y a donc aucune indication d'un assouplissement important et encore moins d'une dissolution, de la limite d'âge à la retraite pour les hommes et très peu pour les femmes. »(Kholi, 2007, p. 264-265, notre traduction).

Un autre exemple est donné par Schumacher, Spoorenberg et Forney à propos de l'âge au départ du foyer parental : « Contrairement à ce que l'hypothèse de la déstandardisation postule, la variabilité de l'âge au départ a diminué à travers les générations » (2006, p. 172).

On a voulu approfondir cette question dans le contexte belge en considérant l'âge à l'entrée et l'âge au départ du marché du travail. L'objectif est de tester l'hypothèse de déstandardisation de l'âge à l'entrée et au départ du marché du travail.

\section{DONNÉES}

Pour tester la validité de cette hypothèse, on a mobilisé les données de l'Enquête socio-économique (ESE) belge (recensement) de 2001 (ESE 2001) $)^{2}$. Celle-ci reprend des questions relatives au dernier diplôme obtenu

2. Ces données portent sur des générations atteignant l'âge légal à la pension en 2001. Elles peuvent sembler vieillies car elles ne permettent pas de prendre en compte les changements récents survenus du fait de la modification de la législation sur les départs du marché du travail (suppression de certaines mesures favorisant les départs anticipés, voir Sanderson, Eggerickx et Burnay, 2012). Malheureusement, le recensement de 2001 est, à ce jour, le dernier réalisé en Belgique. Il aurait été intéressant de disposer de données plus récentes de manière à appréhender les transformations récentes. Toutefois, par rapport à notre objet, il était surtout important de pouvoir couvrir la période sur laquelle porte la déstandardisation, ce que permettent nos données. 
et au moment du départ du marché du travail ce qui n'était pas le cas des recensements précédents ainsi que différentes questions permettant de décrire la situation sociodémographique des individus.

À partir de ces données, on a pu estimer l'âge à l'entrée sur le marché du travail. L'ESE ne livre d'informations ni sur l'âge au premier emploi ni sur le début de la période d'activité des répondants. On a considéré que, pour une majorité d'individus, l'âge de fin d'études coöncide avec l'entrée sur le marché du travail ${ }^{3}$, contrairement au départ du foyer parental qui est de plus en plus souvent différé, le temps pour le jeune de trouver du travail ou de se préparer à vivre de façon autonome (Vandeschrick et Sanderson, 2013). Dès lors, l'âge au dernier diplôme est un choix assez intéressant dans la mesure où l'écart entre l'âge au dernier diplôme et l'âge de fin d'études (non disponible dans le recensement) (tableau 1) est inférieur à 2 ans pour $90 \%$ des personnes ayant terminé leurs études, selon l'Enquête générations et genre (GGP) menée en Belgique.

TABLEAU 1 Écart entre âge de fin d'études et âge au dernier diplôme

\begin{tabular}{|l|c|c|}
\hline Nombre d'années d'écart & Effectif & Proportion (\%) \\
\hline 0 & 6133 & 87 \\
\hline 1 & 245 & 3 \\
\hline 2 & 228 & 3 \\
\hline 3 & 124 & 2 \\
\hline 4 & 80 & 1 \\
\hline 5 à 9 & 127 & 2 \\
\hline 10 et plus & 130 & 2 \\
\hline
\end{tabular}

Source: Enquête générations et genre (GGP)

On a ainsi calculé l'âge moyen à l'obtention du dernier diplôme pour les générations 1904 à 1975. Pour les générations précédentes, lorsqu'on distingue les chiffres selon le sexe, les effectifs de personnes déclarant avoir un diplôme et donnant une date d'obtention ne sont pas en nombre suffisant. Pour les générations les plus jeunes, on a préféré se limiter à celles qui avaient 25 ans révolus (soit celles nées en 1975) au moment du recensement

3. L'âge à l'entrée sur le marché du travail peut différer profondément de l'âge au premier emploi. En effet, l'entrée sur le marché du travail correspond au moment à partir duquel la personne va se définir comme active, qu'elle soit active occupée ou non. Ainsi, l'entrée sur le marché du travail peut se faire aussi bien en tant que demandeur d'emploi (chômeur indemnisé ou non) qu'en tant qu'actif ayant un emploi. 
de manière à éviter, autant que possible, les individus qui seraient encore en formation (Booghmans et collab., 2009). Cela représente des individus ayant quitté l'école entre 1914 et 2001. En Belgique, nous avons vu que l'obligation scolaire a été instaurée en 1914 jusqu'à 14 ans ${ }^{4}$ et prolongée à 18 ans en 1983. Sur l'ensemble des personnes ayant répondu à la question du diplôme et nées en 1904, 70 \% déclarent avoir un diplôme (tableau 2). Progressivement, ces taux vont augmenter et, pour les dernières années, on dépasse les $90 \%$ de diplômés. L'utilisation de l'âge à l'obtention du diplôme pour déterminer l'âge à l'accès au marché du travail présente une limite assez importante pour les générations les plus anciennes. Non seulement la part des personnes sans diplômes est assez importante (environ $30 \%$ ) mais en outre, parmi ceux qui se déclarent titulaires d'un diplôme, $26 \%$ (génération née en 1904) ne peuvent donner de date d'obtention. En privilégiant les personnes ayant un diplôme, on privilégie donc des individus accédant plus tardivement au marché du travail. Toutefois, ce biais vaut essentiellement pour les générations ayant atteint leur sixième anniversaire avant 1914 (date de promulgation de la loi sur l'enseignement obligatoire), soit les générations nées entre 1904 et 1907.

TABLEAU 2 Effectifs par génération des titulaires d'un diplôme

\begin{tabular}{|c|c|c|c|r|r|r|}
\hline \multirow{2}{*}{$\begin{array}{c}\text { Année } \\
\text { de naissance }\end{array}$} & \multicolumn{2}{|c|}{ Effectifs } & \multicolumn{2}{c|}{ Diplômés } & \multicolumn{2}{c|}{$\begin{array}{c}\text { Dont diplômes } \\
\text { avec date }\end{array}$} \\
\cline { 2 - 3 } & Génération & Répondants & \multicolumn{2}{|c|}{} \\
\hline 1904 & 2007 & 1494 & 1042 & $70 \%$ & 774 & $74 \%$ \\
\hline 1905 & 2988 & 2229 & 1595 & $72 \%$ & 1216 & $76 \%$ \\
\hline 1906 & 4072 & 3032 & 2230 & $74 \%$ & 1706 & $77 \%$ \\
\hline 1907 & 5776 & 4435 & 3302 & $74 \%$ & 2516 & $76 \%$ \\
\hline 1908 & 7866 & 6060 & 4639 & $77 \%$ & 3505 & $76 \%$ \\
\hline 1909 & 9741 & 7584 & 5909 & $78 \%$ & 4442 & $75 \%$ \\
\hline 1910 & 12757 & 9984 & 7937 & $79 \%$ & 5939 & $75 \%$ \\
\hline 1911 & 15588 & 12352 & 9985 & $81 \%$ & 7438 & $74 \%$ \\
\hline$\ldots$ & & & & & & \\
\hline 1973 & 136139 & 133335 & 124383 & $93 \%$ & 114301 & $92 \%$ \\
\hline 1974 & 130954 & 128180 & 119508 & $93 \%$ & 109896 & $92 \%$ \\
\hline 1975 & 125548 & 123117 & 114905 & $93 \%$ & 105655 & $92 \%$ \\
\hline
\end{tabular}

Source: Direction générale de la statistique et de l'information économique (DGSIE-ESE, 2001)

4. Toutefois, le travail des enfants de moins de 12 ans est officiellement interdit par la loi dès 1889 . 
À partir des données de l'ESE, on a pu calculer un âge moyen au départ du marché du travail pour chaque génération depuis 1903 (auparavant, les effectifs sont insuffisants pour permettre ce type de calcul, voir tableau 3). Définissant les catégories professionnelles, l'Enquête socio-économique identifie 9 catégories : les étudiants, les personnes ayant un emploi, les demandeurs d'emploi (en 3 catégories), les personnes n'ayant jamais exercé d'activité professionnelle, les pensionnés et les prépensionnés et assimilés. Ce sont ces deux catégories qui ont permis de définir ceux qui ont été considérés comme retraités dans la suite de cet article. Les données utilisées permettent de couvrir 33 générations de retraités :

- depuis celle née en 1903, soit une génération qui débute sur le marché du travail dans l'entre-deux-guerres, au moment où est instauré le système de pension belge à destination des ouvriers et des employés (Sanderson, Eggerickx et Burnay, 2012). Cette génération arrive à l'âge légal à la pension ( 65 ans pour les hommes et 60 ans pour les femmes) entre 1965 et 1970, soit après l'extension du système des retraites aux indépendants et aux professions libérales (loi de 1953), mais surtout avant les chocs pétroliers et la crise des années 1975-1980 et avant le développement des principaux mécanismes de départ anticipé du marché du travail;

- jusqu'à celle née en 1936, soit celle qui passe le cap des 65 ans en 2001, autrement dit la dernière ayant atteint l'âge à la retraite lors de l'ESE.

L'évolution des parcours professionnels et du choix du moment de départ à la retraite est liée notamment à la situation de l'individu sur le marché du travail et à sa position au sein de l'entreprise où il travaille ainsi qu'à certaines variables sociodémographiques comme le sexe (Widmer et Ritschard, 2013). Dans la suite de cet article, l'analyse a ainsi été réalisée en deux temps. Un premier examen a porté sur l'ensemble des retraités en distinguant uniquement les hommes des femmes vu l'évolution fortement différenciée de leur position sur le marché du travail. Par la suite, les analyses ont été poursuivies en tenant compte notamment du niveau d'études, ceci afin de déterminer dans quelle mesure la déstandardisation de l'âge au départ du marché du travail est un mécanisme généralisé ou est propre à certaines catégories de personnes.

Lors de l'ESE, ainsi que cela se faisait déjà pour les recensements précédents, le questionnaire a été auto-administré, d'où un risque d'erreurs de compréhension ou de réponses erronées qu'on ne peut corriger par la suite. Dans le cas présent, les âges à la retraite déclarés couvrent, toutes 
TABLEAU 3 Population par génération âgée de 65 ans et plus selon la situation professionnelle à l'Enquête socio-économique de 2001

\begin{tabular}{|c|c|c|c|c|c|c|c|c|c|c|}
\hline \multirow{2}{*}{$\begin{array}{l}\text { Année de } \\
\text { naissance }\end{array}$} & \multicolumn{2}{|c|}{ Effectif } & \multicolumn{2}{|c|}{$\begin{array}{l}\text { Pensionnés et } \\
\text { prépensionnés }\end{array}$} & \multicolumn{2}{|c|}{ Ne travaille plus } & \multicolumn{2}{|c|}{ En emploi } & \multicolumn{2}{|c|}{ N'ont jamais travaillé } \\
\hline & Hommes & Femmes & Hommes & Femmes & Hommes & Femmes & Hommes & Femmes & Hommes & Femmes \\
\hline 1900 & 19 & 82 & $94,7 \%$ & $82,9 \%$ & $0,0 \%$ & $2,4 \%$ & $0,0 \%$ & $0,0 \%$ & $5,3 \%$ & $14,6 \%$ \\
\hline 1901 & 31 & 175 & $100,0 \%$ & $82,9 \%$ & $0,0 \%$ & $0,6 \%$ & $0,0 \%$ & $0,6 \%$ & $0,0 \%$ & $16,0 \%$ \\
\hline 1902 & 47 & 309 & $100,0 \%$ & $82,2 \%$ & $0,0 \%$ & $2,3 \%$ & $0,0 \%$ & $0,0 \%$ & $0,0 \%$ & $15,5 \%$ \\
\hline 1903 & 89 & 510 & $97,8 \%$ & $80,4 \%$ & $2,2 \%$ & $2,0 \%$ & $0,0 \%$ & $0,0 \%$ & $0,0 \%$ & $17,6 \%$ \\
\hline 1904 & 161 & 741 & $97,5 \%$ & $79,5 \%$ & $1,2 \%$ & $2,0 \%$ & $0,0 \%$ & $0,0 \%$ & $1,2 \%$ & $18,5 \%$ \\
\hline 1905 & 251 & 1184 & $98,8 \%$ & $81,7 \%$ & $0,8 \%$ & $2,4 \%$ & $0,0 \%$ & $0,0 \%$ & $0,4 \%$ & $16,0 \%$ \\
\hline 1906 & 437 & 1730 & $98,4 \%$ & $82,3 \%$ & $0,9 \%$ & $2,0 \%$ & $0,0 \%$ & $0,1 \%$ & $0,7 \%$ & $15,6 \%$ \\
\hline 1907 & 580 & 2433 & $97,1 \%$ & $81,6 \%$ & $1,9 \%$ & $1,9 \%$ & $0,2 \%$ & $0,0 \%$ & $0,9 \%$ & $16,4 \%$ \\
\hline 1908 & 885 & 3508 & $98,4 \%$ & $81,3 \%$ & $0,5 \%$ & $1,7 \%$ & $0,1 \%$ & $0,0 \%$ & $1,0 \%$ & $17,0 \%$ \\
\hline 1909 & 1277 & 4801 & $98,2 \%$ & $83,1 \%$ & $1,1 \%$ & $1,7 \%$ & $0,1 \%$ & $0,0 \%$ & $0,6 \%$ & $15,1 \%$ \\
\hline 1910 & 1784 & 5901 & $98,1 \%$ & $83,8 \%$ & $0,7 \%$ & $1,9 \%$ & $0,2 \%$ & $0,0 \%$ & $1,0 \%$ & $14,3 \%$ \\
\hline \multicolumn{11}{|l|}{$\ldots$} \\
\hline 1934 & 45442 & 50549 & $97,3 \%$ & $83,0 \%$ & $1,1 \%$ & $3,5 \%$ & $1,3 \%$ & $0,5 \%$ & $0,3 \%$ & $13,0 \%$ \\
\hline 1935 & 45980 & 50707 & $97,0 \%$ & $82,7 \%$ & $1,2 \%$ & $3,8 \%$ & $1,5 \%$ & $0,6 \%$ & $0,3 \%$ & $12,9 \%$ \\
\hline 1936 & 45731 & 49813 & $96,6 \%$ & $82,5 \%$ & $1,2 \%$ & $4,0 \%$ & $2,0 \%$ & $0,7 \%$ & $0,3 \%$ & $12,8 \%$ \\
\hline
\end{tabular}

Source: DGSIE-ESE, 2001 
générations confondues, des âges allant de 1 an à 97 ans. Selon les déclarations, $1 \%$ des retraités auraient quitté le marché du travail entre l'âge de 1 an et 19 ans et environ $0,35 \%$ après 69 ans. Pour ces valeurs extrêmes, les effectifs sont faibles. Ce n'est pas le cas pour les femmes déclarant avoir quitté le marché du travail entre 20-29 ans ou 30-39 ans. Elles représentent environ $24 \%$ des retraités, alors que pour les hommes ces chiffres demeurent très bas, moins de $1,5 \%$. Ces résultats peuvent sembler assez surprenants. Il est difficile de déterminer ici s'il s'agit d'une erreur de déclaration. En effet, certaines personnes sont amenées à quitter le marché du travail de manière anticipée (parfois très jeunes) pour des raisons de santé ou, notamment pour la population féminine, pour des raisons liées à la naissance des enfants. Dans la suite de cet article, seules les personnes ayant pris leur retraite après 40 ans $5^{5}$ et avant 70 ans ont été considérées.

TABLEAU 4 Effectif des personnes (âgées de 30 ans ou plus) se déclarant retraitées ou prépensionnées, selon l'âge auquel elles déclarent avoir quitté le marché du travail

\begin{tabular}{|l|c|c|c|c|}
\hline \multirow{2}{*}{ Groupes d'âge } & \multicolumn{2}{|c|}{ Hommes } & \multicolumn{2}{c|}{ Femmes } \\
\cline { 2 - 5 } & Effectifs & Proportion (\%) & Effectifs & Proportion (\%) \\
\hline 1 an-19 ans & 849 & 0,07 & 6802 & 1,25 \\
\hline $20-29$ ans & 3739 & 0,32 & 61568 & 11,35 \\
\hline $30-39$ ans & 12238 & 1,06 & 38969 & 7,18 \\
\hline $40-49$ ans & 135622 & 11,70 & 76011 & 14,01 \\
\hline $50-59$ ans & 707617 & 61,02 & 281553 & 51,89 \\
\hline $60-69$ ans & 291441 & 25,13 & 75232 & 13,86 \\
\hline $70-79$ ans & 6386 & 0,55 & 2233 & 0,41 \\
\hline 80 ans et plus & 1714 & 0,15 & 252 & 0,05 \\
\hline Ensemble & 1159606 & & 542620 & \\
\hline
\end{tabular}

Source: DGSIE-ESE, 2001

\section{ANALYSE ET DISCUSSION}

\section{Âge à l'entrée sur le marché du travail}

L'examen de l'évolution de l'âge moyen à l'obtention du dernier diplôme (figure 1) montre que celui-ci n’a cessé de croître au fil des générations.

5. Le départ à la retraite pouvait être demandé par tout ouvrier mineur de fonds à partir de 20 ou 25 ans de carrière. Sachant que les ouvriers mineurs commençaient leur carrière très tôt, il est probable que certains aient quitté le marché du travail vers 40 ans. 
FIGURE 1 Âge moyen à l'obtention du dernier diplôme

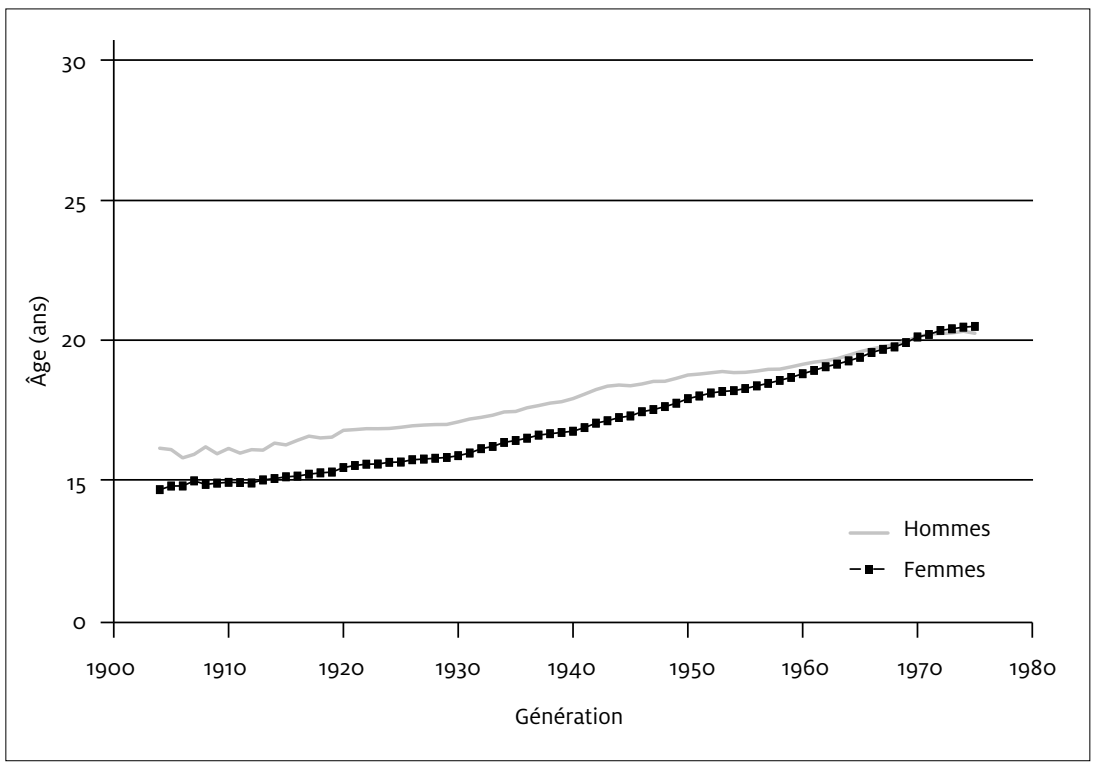

Source: DGSIE-ESE, 2001

Pour la génération la plus ancienne, celle née en 1904, il est de 16,13 ans pour les hommes et de 14,64 ans pour les femmes. Les deux courbes suivent une progression régulière, avec une croissance un peu plus rapide pour les femmes qui progressivement vont combler la différence par rapport aux hommes. Pour la génération 1904, l'écart entre les hommes et les femmes est d'une année et demie en faveur des hommes; pour la génération 1950, cet écart s'est réduit à 0,75 an et pour la génération 1975, le rapport s'est inversé, l'écart est certes minime $(0,25)$ mais il est cette fois en faveur des femmes. Cette évolution va de pair bien évidemment avec l'amélioration de l'accès aux études, leur démocratisation et leur féminisation. Ce premier graphique semble indiquer une nette dissociation entre l'âge maximal de la scolarité obligatoire (14 ans depuis 1914 et 18 ans depuis 1983) et l'âge de fin des études.

En se basant sur l'hypothèse d'une dé-standardisation, on peut s'attendre à ce que l'écart autour de la moyenne augmente. Si on examine la figure 2, qui reprend le coefficient de variation pour les hommes et les femmes, force est de constater qu'il n'en est rien. Au contraire, on observe une diminution de celui-ci à partir des générations nées vers 1940 pour les hommes et vers 1960 pour les femmes. À partir de ces deux générations, la 
FIGURE 2 Évolution l'écart-type autour de la moyenne de l'âge au diplôme

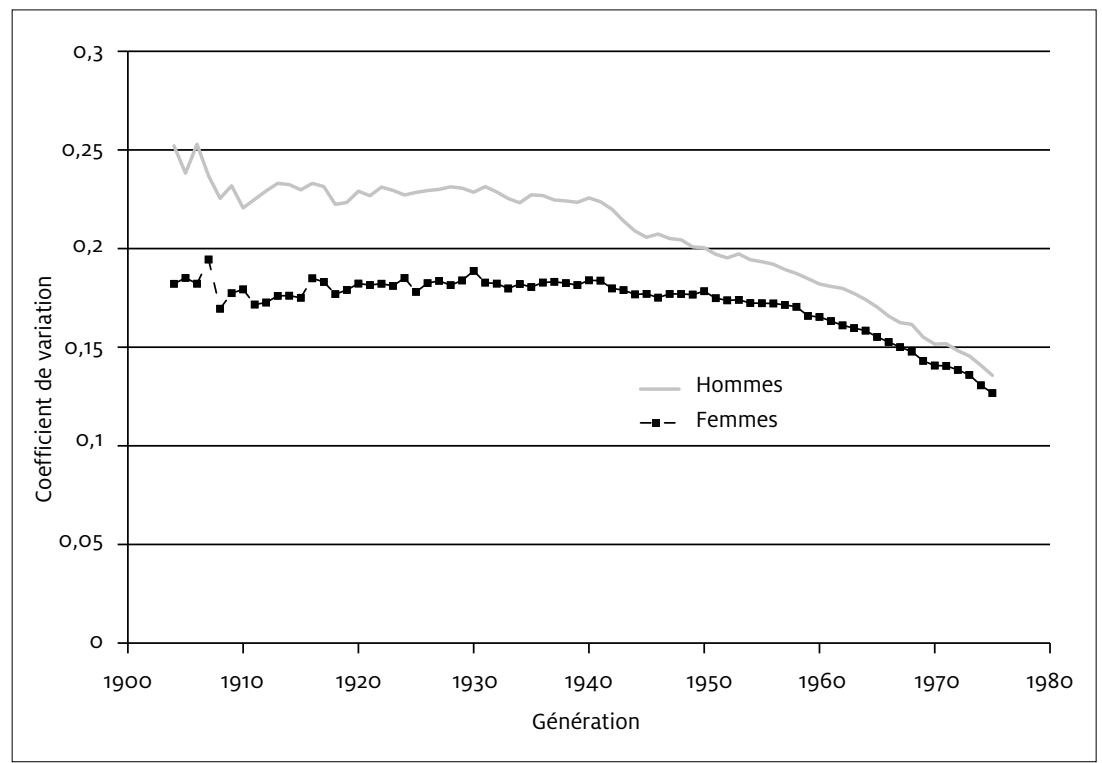

Source: DGSIE-ESE, 2001

tendance est à la diminution des écarts et donc à la standardisation des âges à l'entrée sur le marché du travail. Ainsi, la standardisation qui selon le modèle théorique survient avant les années 1960 (Kholi, 1986) apparaîtrait en fait après cette date. Si on accepte l'utilisation de l'âge au dernier diplôme comme approximation (proxy) de l'âge à l'entrée sur le marché du travail, force est d'admettre qu'il n'y a pas déstandardisation, mais plutôt standardisation tardive. Cette évolution est principalement due à la «massification de l'enseignement», qui a amené de plus en plus de jeunes à rester de plus en plus longtemps aux études, de sorte que progressivement la part des jeunes terminant leur secondaire a augmenté et qu'aujourd'hui le phénomène touche les études supérieures (universitaires ou non) (Navarro, 2012).

\section{Âge au départ du marché du travail}

Un premier constat s'impose : l'âge moyen à la pension (figure 3) des hommes et des femmes n'a cessé de décroître passant de moyennes supérieures à 63 ans pour les hommes nés entre 1903 et 1908 (59 ans pour les femmes) à des moyennes inférieures à 58 ans pour les hommes nés après 1932 (56 ans pour les femmes nées après 1927). Ces résultats sont conformes 
FIGURE 3 Évolution de l'âge moyen au départ du marché du travail

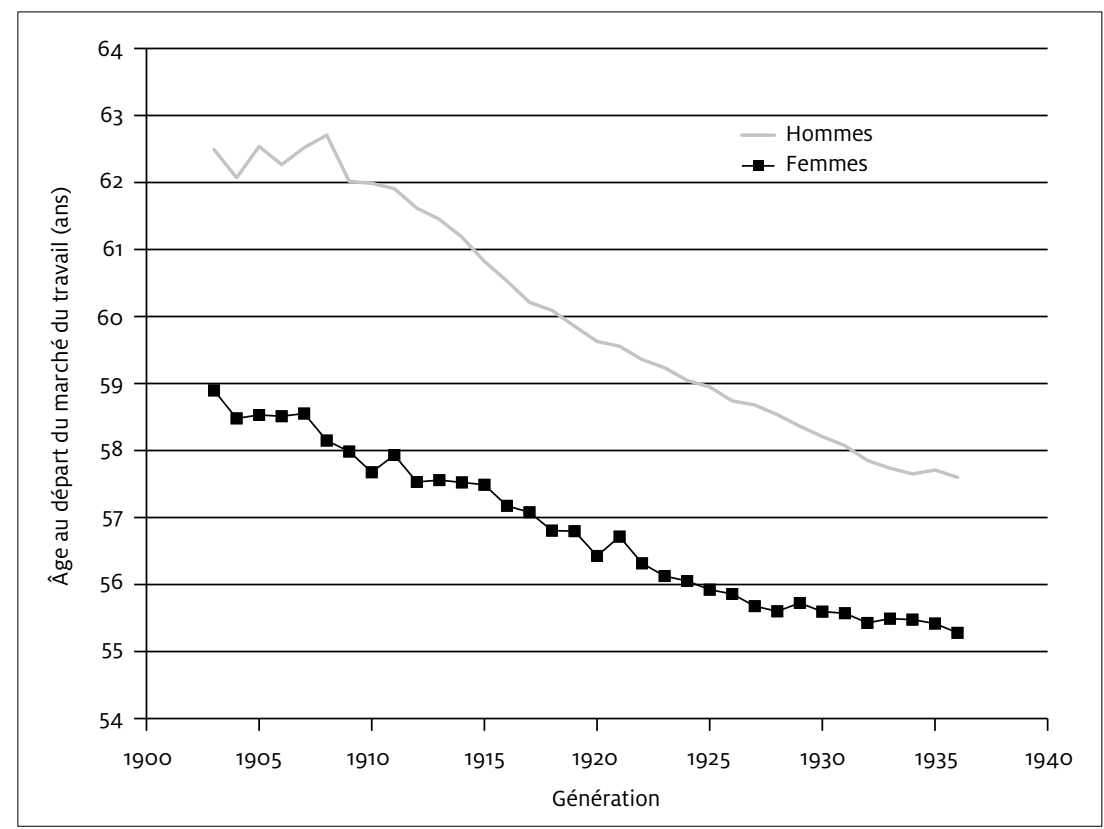

Source: DGSIE-EESE, 2001

à la tendance généralement décrite pour la Belgique et l'Europe en général (Benallah et Mette, 2009), traduisant une anticipation de plus en plus grande de l'âge au départ du marché du travail.

Au-delà de ce premier constat, il s'agissait d'analyser l'évolution du coefficient de variation, indicateur susceptible de traduire un accroissement de la diversification des âges au départ du marché du travail. Les résultats de la figure 4 viennent infirmer notre hypothèse de départ puisqu'on n'observe pas d'accroissement de la variabilité autour de la moyenne si ce n'est en fin de période. Pour les hommes, le coefficient de variation demeure relativement constant, avec à partir de la génération 1925 une légère tendance à la hausse. On quitte alors (soit à partir du milieu des années 1980) le marché du travail en moyenne avant 60 ans. À ce moment-là, les mécanismes de prépension sont, pour la plupart, bien établis et on peut se demander si le léger accroissement observé ne traduit pas les effets d'une déstandardisation qui surviendrait plus tardivement (la littérature fixe le début de la déstandardisation à la fin des années 1960), du moins en ce qui concerne le départ du marché du travail pour les hommes. 
FIGURE 4 Évolution de l'écart-type autour de l'âge moyen au départ du marché du travail

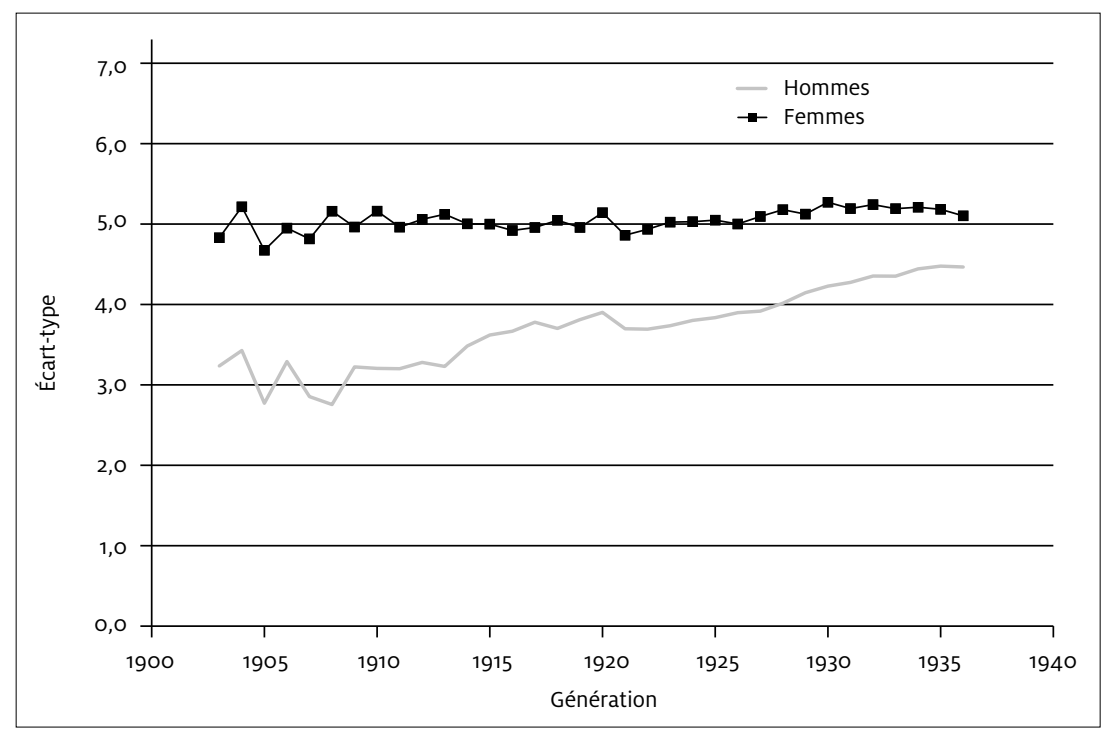

Source: DGSIE-ESE, 2001

Pour les femmes, le coefficient de variation est stable sur l'ensemble de la période d'observation. On ne peut donc pas parler ici de déstandardisation. Ainsi, la théorie soutenant l'hypothèse d'un processus de déstandardisation des parcours de vie à partir des années 1960 semble ne pas se vérifier sur l'âge de transition entre la période d'activité et la retraite.

Avant de pousser plus loin l'interprétation, il paraissait judicieux d'approfondir l'analyse en contrôlant le niveau d'instruction. En effet, à défaut du statut d'occupation et du statut professionnel (non disponible dans les données de l'ESE de 2001 pour les personnes déclarant avoir déjà quitté le marché du travail), le niveau d'instruction peut être utilisé comme approximation (proxy) de ceux-ci, dans la mesure où il les détermine assez largement (Widmer et Ritschard, 2013). En matière d'évolution de l'âge moyen, le déclin est général (figure 5). On observe des différences de niveau : les générations 1905-1909 livrent des valeurs proches de 65 ans pour les hommes disposant d'un diplôme de l'enseignement supérieur, tandis que les valeurs concernant les plus faiblement diplômés (au plus le niveau d'études primaires) sont proches de 63 ans. Cet écart se maintient au fil des générations tandis que les courbes présentent des évolutions parallèles. Les courbes des femmes (figure 6) sont davantage imbriquées, 
FIGURE 5 Évolution de l'âge moyen au départ du marché du travail selon le niveau d'instruction (hommes)

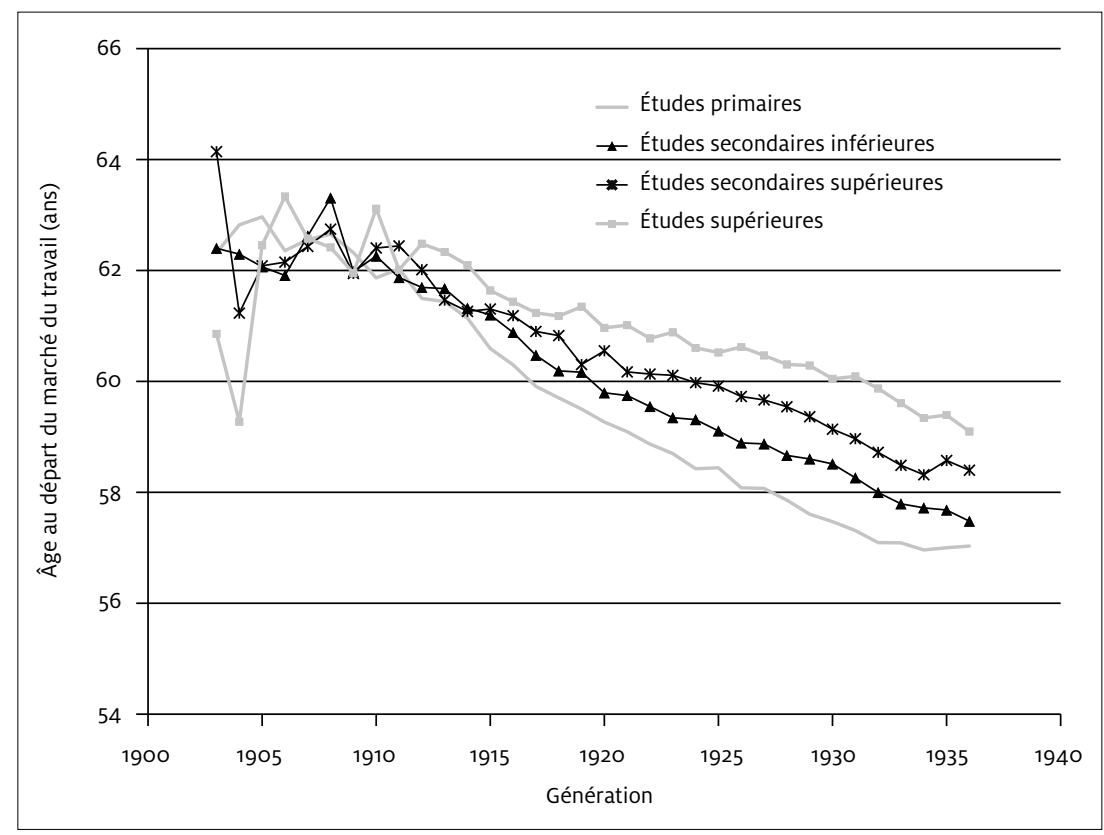

Source: DGSIE-ESE, 2001

notamment en raison de la relative faiblesse des effectifs jusqu'aux générations nées en 1915. Par la suite, on retrouve la même hiérarchie que pour les hommes.

Par contre, le calcul du coefficient de variation (figures 7 et 8 ) met en évidence des évolutions assez différentes. Chez les hommes, il n'y a pas de tendance très claire avant la génération 1920. Selon les courbes, on peut lire une hausse relative mais surtout une convergence de ceux-ci pour atteindre des valeurs proches de 0,08 avec la génération 1920. Par la suite, les écarts vont davantage se creuser. En effet, pour les personnes ayant au plus le niveau d'études primaires, on observe une hausse des écarts qui se poursuit après la génération née en 1922, soit celle arrivant à la pension en 1981 (en moyenne à l'âge de 59 ans). Ce premier groupe voit s'accroître les différences et la variation autour de la moyenne. Dans ce cas précis, on ne peut nier un effet lié à la déstandardisation, ce qui rejoint les constats posés par Widmer et Ritchard (2013) concernant les trajectoires professionnelles qui se complexifient davantage pour les individus peu qualifiés, les autres ayant des parcours plus linéaires. À l'inverse, la situation des personnes 
FIGURE 6 Évolution de l'âge moyen au départ du marché du travail selon le niveau d'instruction (femmes)

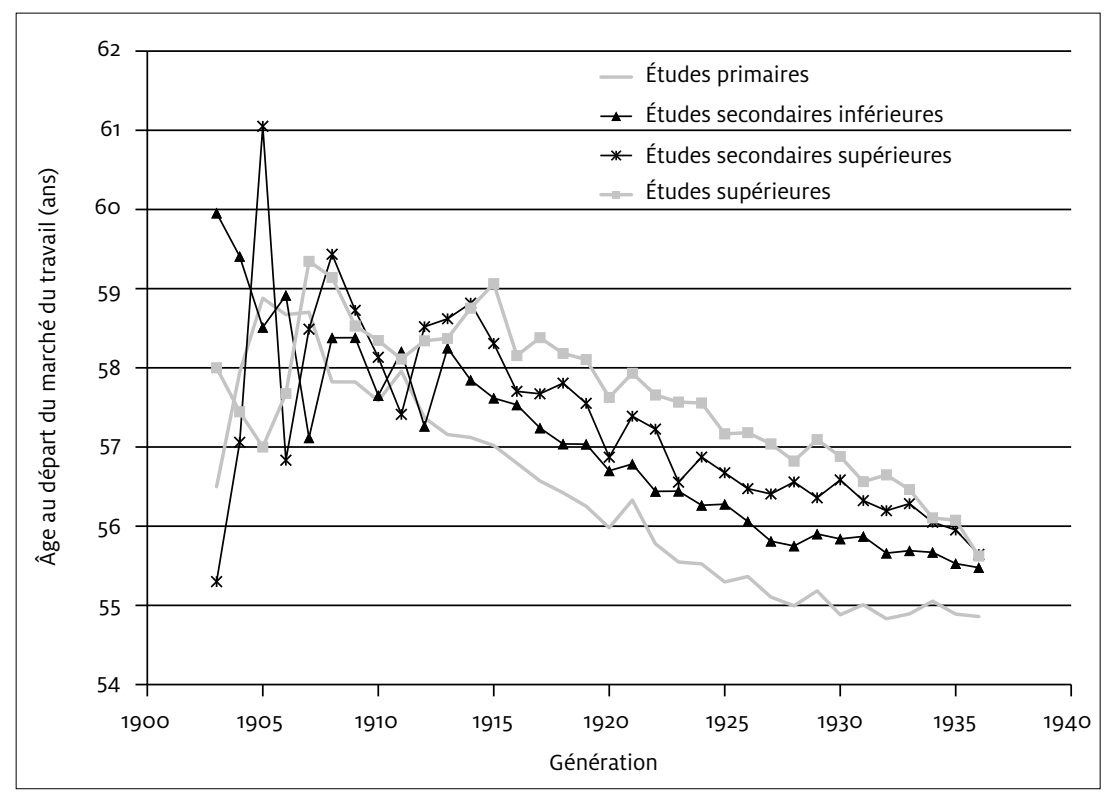

Source: DGSIE-ESE, 2001

ayant un diplôme de l'enseignement supérieur présente beaucoup moins de variations. Après une première baisse entre les générations 1917 et 1922, on assiste à une stabilisation des écarts jusqu'à la dernière génération considérée. Dans ce cas précis, point de déstandardisation mais plutôt une homogénéisation des comportements. Enfin, les deux groupes intermédiaires présentent des situations plus complexes. Après avoir connu une diminution du coefficient de variation et une stabilisation de celui-ci à des niveaux très bas jusqu'à la génération 1930 (quittant le marché du travail à partir de 1988, vers 58 ans), on observe ensuite un accroissement de celui-ci plus marqué pour les individus ayant atteint au plus le diplôme de l'enseignement secondaire inférieur.

Dans cette analyse, le niveau de diplôme apparaît bien comme déterminant pour les hommes. Hormis pour les plus diplômés, on observe une déstandardisation plus ou moins prononcée selon le niveau d'instruction, mais celle-ci serait décalée d'une vingtaine d'années pour les individus ayant obtenu un diplôme de l'enseignement secondaire par rapport au calendrier le plus souvent admis lorsqu'on parle de déstandardisation (Kohli, 1986). 
FIGURE 7 Évolution du coefficient de variation autour de l'âge moyen au départ du marché du travail selon le niveau d'instruction (hommes)

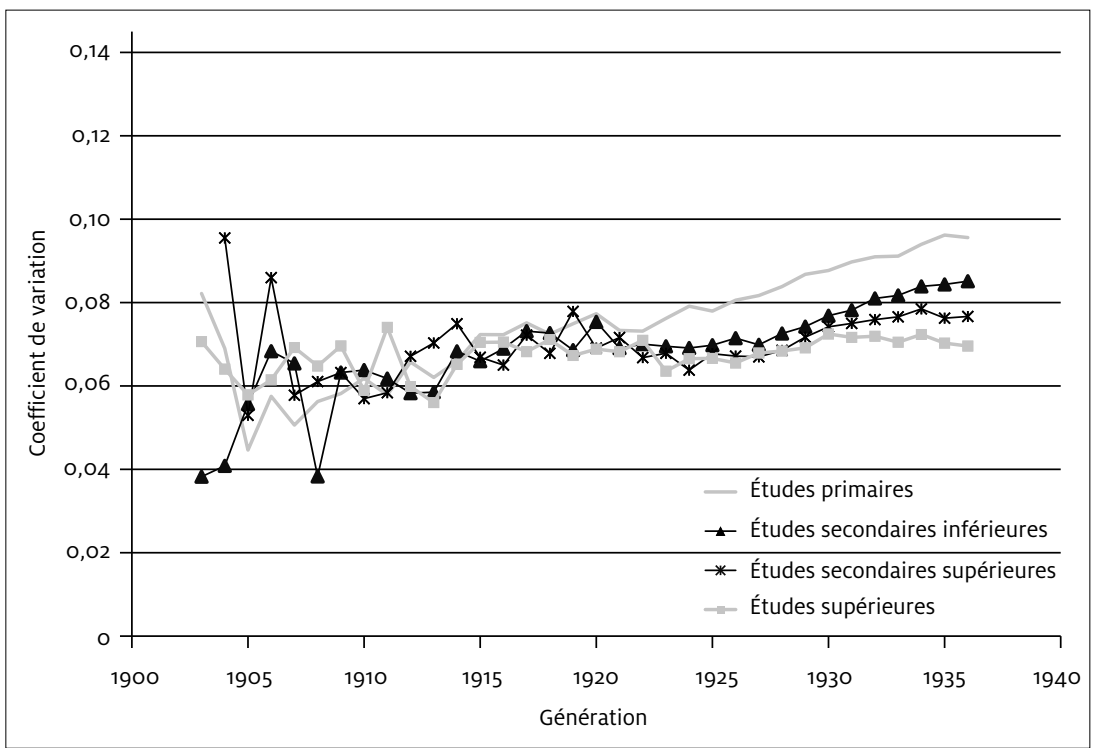

Source: DGSIE-ESE, 2001

FIGURE 8 Évolution du coefficient de variation autour de l'âge moyen au départ du marché du travail selon le niveau d'instruction (femmes)

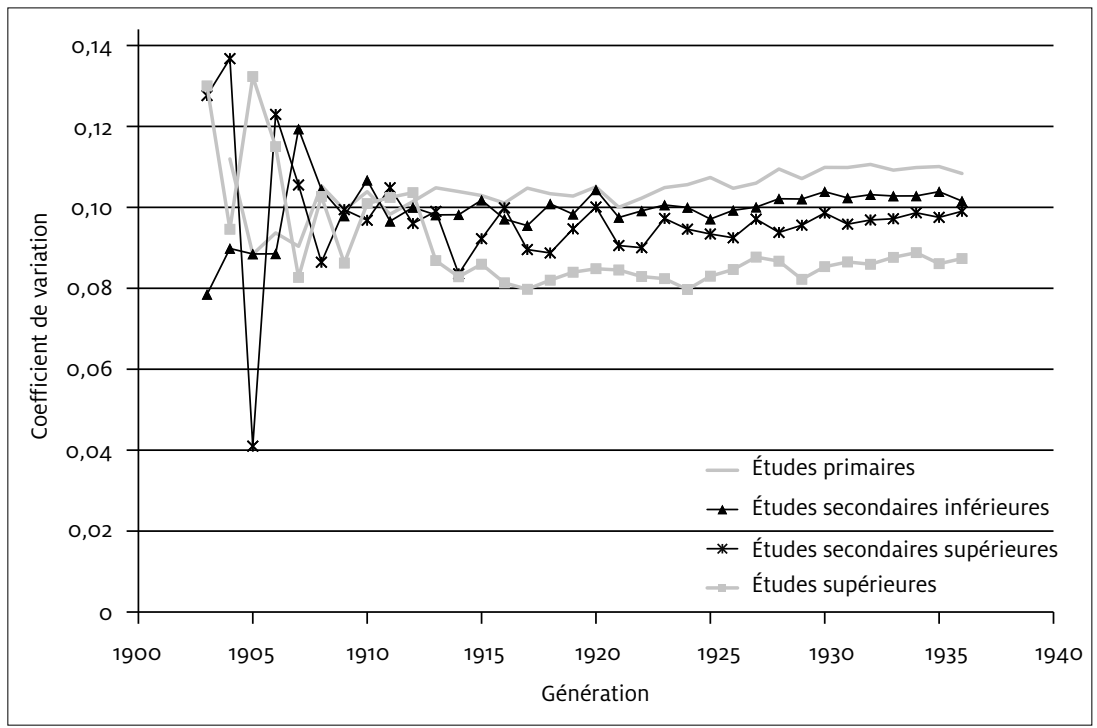

Source: DGSIE-ESE, 2001 
Pour ce qui est de la population féminine, la situation est ici assez différente. Quel que soit le niveau d'étude, on observe un maintien généralisé du coefficient de variation qui se poursuit jusqu'aux dernières générations considérées. Les valeurs sont plus élevées que ce qui est observé pour la population masculine. Seules les femmes les plus diplômées semblent enregistrer une baisse de la variation qui les amène dès la génération née en 1915 à des valeurs comparables à celles observées pour les hommes. On peut ici conclure qu'en ce qui concerne la population féminine il n'y a aucun effet de déstandardisation lié au niveau d'études, bien que dans le même temps l'ensemble de la population féminine voit diminuer l'âge moyen au départ du marché du travail.

\section{CONCLUSION}

L'objectif de cet article était de tester l'hypothèse d'une déstandardisation des âges à l'entrée et au retrait du marché du travail. L'examen de la littérature met en évidence une situation complexe où certains éléments confirment cette hypothèse et d'autres l'infirment. L'analyse de l'âge moyen à l'entrée sur le marché du travail montre dans le cas belge, une diminution des écarts autour de la moyenne qui se marque principalement à partir des générations nées en 1940, soit entrant sur le marché du travail après les années 1960. De ce point de vue, l'hypothèse d'une déstandardisation des âges à l'entrée sur le marché du travail est infirmée. Au contraire, on assisterait à une standardisation tardive des âges.

L'examen de l'âge au départ du marché du travail à partir des données belges met en évidence une diminution régulière de celui-ci tant pour les hommes que pour les femmes traduisant l'accroissement des départs anticipés. Toutefois, pour ce qui est du coefficient de variation, ils ne varient guère pour les hommes jusqu'à la génération 1930 (arrivant à la retraite au début des années 1990) lorsqu'ils prennent une tendance légèrement à la hausse et diminuent pour les femmes pour l'ensemble des générations. Ce second résultat infirme partiellement l'hypothèse de travail. Pour les hommes, la déstandardisation surviendrait plus tard que prévu tandis que pour les femmes, elle ne se marquerait pas.

En contrôlant le niveau d'études des personnes quittant le marché du travail, on constate que, quel que soit le niveau d'étude, au fil des générations, la tendance générale est à la diminution de l'âge au départ du marché du travail. Par contre, on observe que, si le déclin est généralisé, le coefficient de variation augmente pour les hommes les moins diplômés alors qu'il diminue pour les hommes disposant d'un diplôme de l'enseignement 
supérieur. Ainsi, on observe bien pour certaines catégories (les hommes les moins diplômés) un effet de déstandardisation. Pour ce qui est de la population féminine, la situation est différente en ce sens où, quel que soit le niveau de diplôme, il n'y a pas d'effet de déstandardisation, le coefficient de variation n'augmentant en aucune façon.

Ces résultats confirment ceux obtenus par ailleurs (Brückner et Mayer, 2005; Kholi, 2007; Schumacher, Spoorenberg et Forney, 2006). Il semble qu'on ne peut pas parler aujourd'hui d'une déstandardisation des âges auxquels l'entrée et la sortie du marché du travail sont vécues. On peut dès lors se demander s'il faut abandonner l'hypothèse d'une déstandardisation des âges ou la reformuler. En effet, dans cet article, on a examiné la situation de deux âges institutionnalisés (l'âge de fin d'études, partiellement institutionnalisé au travers de l'âge d'obligation scolaire et l'âge au départ du marché du travail fixé par l'âge légal à la retraite). La question reste néanmoins ouverte pour des événements où le libre arbitre joue davantage : l'âge à la mise en union, l'âge au premier enfant, l'âge au départ du foyer parental... soit des âges non fixés par la loi, non institutionnalisés.

\section{BIBLIOGRAPHIE}

Aubert, P. 2012. «Les âges de sortie d'activité », Revue française des affaires sociales, $4: 79-83$.

Benallah, S. et C. Mette. 2009. "Âge moyen de départ à la retraite : tendances récentes et évolution attendues ", Retraite et société, $58: 166-183$.

Bessin, M. 1999. "La compression du temps : une déritualisation des parcours de vie », Les âges de la vie, Éducation permanente, $138: 75-85$.

Booghmans, M., V. Cortese, P. Desmarez, M. Hamzaoui, R. Loyen, E. Stevens, P. van der Hallen, A. Valkeneers, M. Veinstein et C. Vermandere. 2009. Scolarité et emploi, Monographie de l'enquête socio-économique générale de 2001. Bruxelles, Direction générale de la Statistique économique, $214 \mathrm{p}$.

BRÜCKNER, H. et K. U. MAYER. 2005. «De-standardization of the life course : what it might mean? And if it means anything, whether it actually took place?", Advances in Life Course Research, 9:27-53.

Burnay, N. 2002. "Retrait anticipé du marché de l'emploi et exclusion sociale ", Gérontologie et société, 102 : 109-125.

Carpentier, N. et D. White. 2013. « Perspective des parcours de vie et sociologie de l'individuation ", Sociologie et sociétés, 45, 1:279-300.

Cavalli, S. 2007. « Modèle de parcours de vie et individualisation », Gérontologie et société, 123: 55-69.

ELDER, G. 1998. Children of the great depression: social change and life experience. New York, Westview Press, $470 \mathrm{p}$. 
Elder, G. H., J. M. Kirkpatrick et R. Crosnoe. 2003. "The Emergence and Development of Life Course Theory", dans J. T. Mortimer et M. J. SHANAKAN (dir.), Handbook of the Life Course, New York : 3-19.

Feller, E. 2005. Histoire de la vieillesse en France, 1900-1960. Du vieillard au retraité. Paris, Seli Arslam, 352 p.

Gaudet, S. et M. Turcotte. 2013. "Sommes-nous égaux devant l'“injonction” à participer? Analyse des ressources et des opportunités au cours de la vie ", Sociologie et sociétés, $45: 117-145$.

Generation And gender program. Enquête Générations et genres [en ligne]. GGP Belgium. http ://www.ggps.be/survey_fr.htm

Guillemard, A.-M. 2004. "Intégrer la perspective du cycle de vie dans l'analyse de la protection sociale ", Contribution à la session du RTF6 du $1^{\text {er }}$ congrès de l'AFS, 24 février.

Hicks, P. 2013. «L'analyse sociale au sein d'une société habilitante : pertinence des perspectives fondées sur les parcours de vie dans l'élaboration des politiques ", Sociologie et sociétés, $45:$ 301-333.

Koнli, M. 1986. "The world we forgot : a historical review of life course ", dans V. W. Marshall (dir.), Later life. The social psychology of aging, Londres : 271-303.

KoHLI, M. 2007. «The Institutionalization of the Life Course : Looking Back to Look Ahead ", Research in human development, 4: 253-271.

Lesthaeghe, R. et K. Neels. 2002. "From the First to the Second Demographic Transition - An Interpretation of the Spatial Continuity of Demographic Innovation in France, Belgium and Switzerland », European Journal of Population, $18,4: 225-260$.

Loriaux, F. 1999. Enfants-machines. Histoire du travail des enfants en Belgique aux $19^{e}$ et $20^{e}$ siècles. Bruxelles, $128 \mathrm{p}$.

Macmillan, R. 2005. "The structure of the life course : classic issues and current controversies ", Advances in life course research, 9: 3-24.

Moulaert, T. 2006. "Le Pacte de solidarité entre les générations », Courrier hebdomadaire du CRISP, 1906-1907 : 5-68.

Navarro, M. 2012. "Massification de l'enseignement, inégalités d'apprentissage et pratiques pédagogiques", Regards croisés sur l'économie: 203-207.

OrIs, M. 2006. Intimité familiale et fécondité des familles. Jalons historiques à travers une démographie sociale, Working paper of the Scientific Research Community Historical Demography, 3, $20 \mathrm{p}$.

Roussel, L. et A. Girard. 1982. "Régimes démographiques et âges de la vie ", dans Les âges de la vie, Actes du VIIe colloque National de Démographie, Tome 1, Travaux et documents de l'INED, $96: 15-23$.

Royer, D. 2002. "Qu'en est-il de la « valeur travail » dans notre société contemporaine? », Empan, $46: 18-25$.

Sanderson, J.-P., T. Eggerickx et N. Burnay. 2012. «Vieillissement de la population et mise en place du système des retraites en Belgique ", communication présentée au colloque international de l'AIDELF, 12-16 novembre 2012. 
SAPIn, M., D. Spini et E. Widmer. 2007. Les parcours de vie. De l'adolescence au grand âge. Lausanne.

Schumacher, R., T. Spoorenberg et Y. Forney. 2006. "Dé-standardisation, différenciation régionale et changements générationnels. Départ du foyer parental et modes de vie en Suisse au $\mathrm{xx}^{\mathrm{e}}$ siècle ", Revue européenne de démographie, 22, 2 : 153-177.

Vander Stricht, V. 1997. Les taux de chômage en Wallonie, Discussion Paper $d u$ Service de la Statistique du Ministère de la Région wallonne, 9707, 36 p.

Vandeschrick, C. et J. P. Sanderson. 2013. Départ du nid familial. Des constances et des contrastes. GGP-Policy Brief, 5 .

Vanthemsche, G. 1994. La sécurité sociale. Les origines du système belge. Le présent face à son passé. Bruxelles, Deboeck.

Widmer, E., G. Ritschard et N. Müller. 2009. «Trajectoires professionnelles et familiales en Suisse : quelle pluralisation?", dans M. Oris et collab. (dir.), Transitions dans les parcours de vie et construction des inégalités, Lausanne, Presses polytechniques et universitaires romandes : 253-272.

Widmer, E. D. et G. Ritschard. 2013. «Tous égaux devant la pluralisation des parcours de vie? Déstandardisation des trajectoires familiales et professionnelles et insertions sociales ", dans S. GAudet, N. Burlone et M. LÉvesque (dir.), Repenser la famille et ses transitions. Repenser les politiques publiques, Presses de l'Université de Laval : 79-110.

\section{ABSTRACT \\ Jean-Paul SANDERSON \\ Life course and ages of transitions in Belgium: Towards a de-standardization of ages at entry and exit from the labor market?}

Recent decades have witnessed profound changes in the pattern of working lives. Entry into professional activity has become increasingly late and complex, while exit from the labor market has tended to occur much earlier in Europe and particularly in Belgium, to the extent that policy makers have been prompted to intervene. Analysis of this double transformation has led to formulation of a hypothesis of "life-course de-standardization" starting in the 1960 . Using retrospective data for Belgium, this article aims to test this hypothesis of de-standardization by examining ages at entry to and exit from the labor market between 1965 and 2001. 